\title{
LA PRESENCIA PEDAgógiCA EN LA CONSTRUCCIÓN DE LA RELACIÓN EDUCATIVA. EL CASO DE UNA MAESTRA DE EDUCACIÓN PRIMARIA
}

\author{
The pedagogical presence in the construction \\ of the educational relationship. \\ The case of a Primary School teacher
}

Diego MARTÍN-ALONSO, Nieves BLANCO y Eduardo SIERRA

Universidad de Málaga. España.

diegomartin@uma.es; nblanco@uma.es; esierra@uma.es

bttps://orcid.org/0000-0001-7367-7862; https://orcid.org/0000-0001-7735-4593;

bttps://orcid.org/0000-0002-9925-1656

Fecha de recepción:08/06/2020

Fecha de aceptación: 30/08/2020

Fecha de publicación en línea: 01/11/2020

Cómo citar este artículo: Martín-Alonso, D., Blanco, N., y Sierra, E. (2021). La presencia pedagógica en la construcción de la relación educativa. El caso de una maestra de Educación Primaria. Teoría de la Educación. Revista Interuniversitaria, 33(1), 111-131. https://doi.org/10.14201/teri.23389

RESUMEN

La experiencia de relación no se deja definir plenamente —en un sentido positivista- porque su naturaleza es inefable, por lo que resulta aventurado pensar la relación solo desde un punto de vista analítico. Aunque ese trabajo descriptivo y de conceptualización es imprescindible, también es necesaria una mirada integral a la experiencia de relación y las cualidades que hacen que esta sea educativa. Este es el propósito del texto: pensar de una manera integral, holística, sobre el lugar simbólico 
DIEGO MARTÍN-ALONSO, NIEVES BLANCO Y EDUARDO SIERRA LA PRESENCIA PEDAGÓGICA EN LA CONSTRUCCIÓN DE LA RELACIÓN EDUCATIVA. EL CASO DE UNA MAESTRA DE EDUCACIÓN PRIMARIA

y físico del docente en la relación educativa. Esta tarea la realizamos a través de la noción de presencia y pensando con la experiencia de dos alumnas de educación primara a las que hemos acompañado durante dos cursos escolares. Siguiendo las propuestas de la fenomenología hermenéutica, los procesos de investigación han sido la observación de cerca y las conversaciones hermenéuticas. A partir de este trabajo de campo, iniciamos un diálogo con estudios sobre la presencia, la pedagogía de la presencia y la narración de experiencias concretas que hemos vivido junto a las alumnas; de modo que este diálogo nos permita vislumbrar algunos aspectos estructurales de la relación educativa. En concreto en las experiencias que hemos estudiado hemos identificado tres gestos que nombramos como: invitar, estar y retirarse. Estos gestos nos ayudan a pensar en las cualidades del oficio docente y la relación educativa, anclados en las características de este acontecimiento particular y conscientes de su singularidad, evitando la tentación de pretender generalizarlas o extrapolarlas a otras relaciones educativas.

Palabras clave: presencia; fenomenología; relación alumno-maestro; filosofía de la educación; enseñanza.

\section{ABSTRACT}

It is not possible to fully define the relationship experience -in a positivist way- because its nature is ineffable, so it is risky to think about the educational relationship only from an analytical point of view. Although this descriptive and conceptualization work is essential, it is also necessary a comprehensive look at the relationship experience and the qualities that make it educational. That is the purpose of this text: to think holistically about the symbolic and physical place of the teacher in the educational relationship. This task is carried out through the notion of presence and thinking with the experience of two primary education students whom we have accompanied during two school years. Following the proposals of hermeneutic phenomenology, the research processes have been close-observation and hermeneutic conversations. Based on this fieldwork, we began a dialogue with studies on presence, the pedagogy of presence and the narration of concrete experiences that we have lived with the students. We pretend that this dialogue allows us to glimpse some structural aspects of the educational relationship. Specifically, in the experiences we have studied we have identified three gestures that we name as: invite, be and leave. These gestures help us think about the qualities of the teaching profession and the educational relationship, anchored in the characteristics of this particular event and aware of its uniqueness, avoiding the temptation to try to generalize or extrapolate them to other educational relationships.

Key words: presence; phenomenology; student-teacher relationship; philosophy of education; teaching. 
DIEGO MARTÍN-ALONSO, NIEVES BLANCO Y EDUARDO SIERRA

LA PRESENCIA PEDAGÓGICA EN LA CONSTRUCCIÓN DE LA RELACIÓN EDUCATIVA.

EL CASO DE UNA MAESTRA DE EDUCACIÓN PRIMARIA

\section{INTRODUCCIÓN}

Lo que el alumnado aprende, como nos dice Clandinin (2013), no es lo que se planifica (el programa de estudios) sino lo que experimenta en el aula en tanto que currículum vivido (Martín-Alonso, 2019; Pinar e Irwin, 2004). Esto es, el currículum se construye en la experiencia del alumnado en el aula en relación con las compañeras, compañeros, el profesorado y la cultura.

La relación educativa se constituye, desde este enfoque, como un aspecto estructural de los estudios curriculares y la teoría educativa (Vila, 2019; Arbiol, 2018). Es por esto que, desde diversos enfoques metodológicos y epistemológicos, se vienen realizando investigaciones que tratan de estudiar, vislumbrar, las cualidades de la relación educativa y las disposiciones docentes que la sostienen (Bárcena, 2018; Martín-Alonso, 2019; Piussi y Mañeru, 2006; Jordán, 2011; Arnaus, 2013; Recalcati, 2014, 2016; Van Manen, 1998).

Así, en el afán por analizar y comprender la relación educativa, tratamos de identificar cualidades que nos permitan conocer su naturaleza. Pero si la relación educativa es la experiencia del encuentro entre docente y estudiante (Cima, 2012), y la experiencia es inefable ${ }^{1}$, debemos aceptar que ese conocimiento será siempre aproximativo y que habrá aspectos que permanecerán ocultos o en penumbra (Van Manen, 1998, 2003).

La relación educativa no se construye, entonces, a través de un conjunto de procedimientos que se aplican sistemáticamente o en un orden secuencial. Se trata más bien de un actuar del docente, que busca el encuentro con el niño o niña, y que, para poder entender, los pedagogos desgranamos en dimensiones o cualidades. La experiencia de relación no se deja definir, desvelar plenamente (en un sentido positivista), porque su naturaleza no es fragmentable, por lo que resulta aventurado pensar la relación solo desde un punto de vista analítico. Aunque ese trabajo descriptivo y de conceptualización es imprescindible, también es necesaria una mirada integral a la experiencia de relación y las cualidades que hacen que esta sea educativa.

Bárcena (2012) nos invita a realizar esta mirada no fragmentada a la relación educativa en términos de presencia. En concreto, en su texto «Una pedagogía de la presencia. Crítica filosófica a la impostura pedagógica» nos dirá que:

Lo más interesante de toda relación de aprendizaje es, precisamente, ese 'hacernos presentes en lo que hacemos', esa relación cara-a-cara, en lo que enseñamos y en lo que aprendemos. La aludida pedagogía de la presencia tiene aquí claramente su máxima relevancia: ¿Cómo nos hacemos presentes, cuando enseñamos y cuando aprendemos, en eso que nos pasa al transmitir y al recibir, al enseñar y al tratar de comprender, y siempre en relación al objeto que media en la relación profesor-alumno? (p. 54).

1. En tanto que la experiencia no se deja atrapar en objetivos o de manera analítica, ni es de naturaleza lingüística, entendemos siguiendo a Van Manen (2003) que esta es inefable. 
DIEGO MARTÍN-ALONSO, NIEVES BLANCO Y EDUARDO SIERRA LA PRESENCIA PEDAGÓGICA EN LA CONSTRUCCIÓN DE LA RELACIÓN EDUCATIVA. EL CASO DE UNA MAESTRA DE EDUCACIÓN PRIMARIA

¿Cómo «hacernos presentes en lo que hacemos»? ¿Qué lugar ocupa el docente en la experiencia de cada estudiante en el aula? ¿Y en su historia de vida? ¿Siempre tiene la misma presencia? ¿Debe retirarse? ¿Cuándo? En definitiva ¿cuál es la presencia del docente en la vida del estudiante? De estas preguntas de investigación aparece el foco de nuestro texto: ${ }^{2}$ pensar sobre el lugar simbólico (y también físico) del docente en la relación pedagógica.

Nuestro propósito, entonces, es estudiar las cualidades que la literatura especializada define como inherentes a la relación educativa. Pero no para tecnificarlas o crear una suerte de inventario de saberes docentes, sino para comprenderlas de manera global, mirando a los gestos (físicos, didácticos, pedagógicos y simbólicos) del docente y cómo a través de ellos se hace presente en la experiencia del alumnado y se construye la relación educativa.

El estudio de la relación educativa, tal como la vamos entendiendo, se puede abordar solo desde situaciones concretas. Por ello nos centramos en la experiencia de dos alumnas de educación primaria, Alisha y Hanna ${ }^{3}$, a las que hemos acompañado durante dos cursos escolares (2017/18 y 2019/20). Desde el marco de la fenomenología hermenéutica y su pertinencia para el estudio de la relación educativa (Ayala, 2018), hemos realizado con posterioridad al trabajo de campo lo que Van Manen (2003) denomina un análisis exegético y mediante ejemplos. Esto es, hemos puesto en diálogo los textos de campo con el trabajo de otros autores para pensar en el lugar que ocupa una maestra en la experiencia educativa de dos de sus alumnas.

En concreto en las experiencias que hemos estudiado hemos identificado tres gestos que nombramos como: invitar, estar y retirarse. Estos gestos nos ayudan a pensar en las cualidades del oficio docente y la relación educativa, anclados en las características de este acontecimiento particular y conscientes de su singularidad, evitando la tentación de pretender generalizarlas o extrapolarlas a otras relaciones educativas.

\section{MARCO TEÓRICO}

\section{1. ¿Por qué investigar sobre la presencia en pedagogía?}

Desde el paradigma hegemónico del positivismo, dice Bárcena (2012), se ha desdeñado en la tradición de la filosofía y la teoría de la educación el estudio sobre la presencia del docente en la relación educativa. Así, los estudios educativos se han centrado tradicionalmente en el aprendizaje individual que hace el estudiante, algo

2. Este artículo se enmarca en el proyecto de investigación: Relaciones educativas y creación del currículum: entre la experiencia escolar y la formación inicial del profesorado. Indagaciones narrativas (EDU2016-77576-P), Plan Nacional de I+D+i del Ministerio de Economía y Competitividad.

3. Todos los datos que puedan identificar a las personas junto a las que hemos investigado han sido modificados para garantizar su anonimato. 
que se ha acentuado en las últimas décadas con el establecimiento de la meritocracia y la competitividad como valores sociales y económicos primarios; dando lugar al fenómeno que se ha denominado como «learnification» (Biesta, 2009). Esto es, la tendencia de la pedagogía a desplazar la atención desde la enseñanza al aprendizaje, donde pierde centralidad la relación docente-estudiante y la presencia física y simbólica de los cuerpos en relación, que es la que en última instancia permite unos aprendizajes concretos.

Nos encontramos así, desde el comienzo de siglo XXI, en la «era del aprendizaje», una época en la que el "lenguaje del aprendizaje» es omnipresente en la política y los debates educativos (Biesta, 2017), pretendiendo sustituir al lenguaje de la educación. Esto se puede observar, por ejemplo, en la actual concepción que se tiene del docente en algunas teorizaciones como facilitador del aprendizaje, o en los cambios de denominación de algunos términos educativos (Biesta, 2011).

Como consecuencia de esta aprendificación, al poner el foco principalmente en el aprendizaje se deja de lado la experiencia de relación, como si esta fuese secundaria; o como si el estudiante aprendiese independientemente de cuál sea la experiencia de relación y, en definitiva, de la experiencia vivida en el aula. Se nos antoja necesario, en este contexto, centrar los estudios pedagógicos no en el aprendizaje, sino en la enseñanza, diferenciando entre «aprender de» $\mathrm{y}$ «ser enseñado por» (Biesta, 2017).

En definitiva, se trata de estudiar cómo en la experiencia de relación con el docente se va recreando el proceso de subjetivación. Al hablar de subjetivación nos referimos a la idea de Biesta (2017), a la condición de sujeto de los estudiantes y el proceso - inacabable- de llegar a ser y sentirse un sujeto independiente, esto es, libre y con ello responsable de la propia acción.

En la investigación educativa debemos, por tanto, dirigir la atención no solo al proceso de aprendizaje, sino también a la relación educativa y cómo se va desenvolviendo el docente en esa experiencia. Esto nos lleva a pensar en el encuentro docente-estudiante como el eje del proceso de subjetivación y en lo que podríamos llamar como una "pedagogía de la presencia" (Bárcena, 2012). Pensar en una pedagogía de la presencia significa situar en el centro del proceso educativo no al estudiante, ni los programas curriculares, sino la experiencia de relación educativa. Significa entender que es la experiencia del encuentro con el docente la que media en el citado proceso de subjetivación.

\subsection{Una aproximación a la noción de presencia}

Adultos y niños experimentamos el mundo desde nuestra subjetividad. Cuando hablo o interactúo con los demás, dice Van Manen (1998), "soy constantemente el sujeto de mi discurso y de mis acciones: creo, veo, siento, oigo, entiendo, amo, hago, juego, me pregunto cosas». (p. 150). Sin embargo, en esta primacía del yo, aparece la experiencia de la alteridad, experimentamos la presencia del otro, otra 
DIEGO MARTÍN-ALONSO, NIEVES BLANCO Y EDUARDO SIERRA LA PRESENCIA PEDAGÓGICA EN LA CONSTRUCCIÓN DE LA RELACIÓN EDUCATIVA. EL CASO DE UNA MAESTRA DE EDUCACIÓN PRIMARIA

persona que aparece en nuestra experiencia en un lugar concreto de nuestra historia de vida: ¿Cómo se hace presente? ¿Por qué sentimos esa presencia? ¿Cuál es la naturaleza de la presencia de una maestra? ¿Qué hace que la vivamos como una maestra y no otra cosa?

Rodgers y Raider-Roth (2006) introducen una primera definición de presencia que puede orientarnos al pensar en estas preguntas: "Hacerse presente es entrar en relación, en conexión, con los estudiantes, sus aprendizajes, el conocimiento y uno mismo». (p. 284). ${ }^{4}$ Esta primera definición, aunque escueta, nos señala tres términos que parecen esenciales para comprender cómo el docente se hace presente: conexión, estudiante y conocimiento. Hablar de presencia en la enseñanza alude así a esa experiencia en que el docente percibe y es capaz de responder a las necesidades del alumnado, acercándose a lo que Van Manen (1998) llama comprensión pedagógica. Es significativo en este sentido el estudio de Molina, Blanco y García (2013), que señala cómo los estudiantes con historias de éxito escolar reconocen a aquellos docentes que los han escuchado, que se han acercado a comprender sus necesidades.

Ahora bien, en esta tarea el docente se encuentra con una dificultad que debemos considerar. En el escenario profesional docente (Clandinin y Connelly, 1995) podemos identificar dos espacios: dentro y fuera del aula. En el tránsito entre uno y otro espacio los docentes son objeto de presiones, indicaciones, marcos normativos, etc., que les dicen lo que debe hacer, de manera que al llegar al aula se encuentran con tensiones entre lo que demandan los stakeholders y lo que desde su juicio pedagógico entienden más oportuno (Martín-Alonso, Blanco y Sierra, 2018). Ante estas tensiones el docente se puede ver movido a actuar de manera artificial, generando una disociación con su propio sentir personal y profesional, haciendo difícil así que se muestre realmente presente en el aula: "la aparente falta de continuidad entre los dos mundos de la maestra [personal y profesional] puede convertirse en angustiante y su capacidad para estar presente verse comprometida". (Rodgers y Raider-Roth, 2006, p. 272).

Por otro lado, desde diversos enfoques (Bárcena y Mèlich, 2000; Clandinin, 2013; Contreras, 2016) se señala que la subjetividad tiene una naturaleza narrativa. Esto es, necesitamos contarnos historias, relatarnos lo vivido para darle sentido y, en última instancia, constituirnos como sujetos. Estas historias tienen, en consecuencia, una naturaleza lingüística, se constituyen a través de la palabra (Clandinin, 2013; Martín-Alonso, 2019). Ahora bien, la palabra nos antecede, nuestra propia palabra es anterior a nuestra llegada al mundo. Entonces, el sujeto es producto de la palabra del otro. No existe el sujeto autónomo, sino el sujeto tomado por la palabra del otro; en definitiva, el proceso de subjetivación está construido por el discurso del otro (Recalcati, 2014, 2016).

4. Todas las traducciones del texto son propias. 

EL CASO DE UNA MAESTRA DE EDUCACIÓN PRIMARIA

Esto no significa que seamos determinación absoluta, que vivamos en una dependencia plena del otro en la subjetivación. Existiendo la determinación subjetiva, hay espacio también para la decisión de cada sujeto acerca de qué hacer con aquello que lo determina. Esto es, la historia de cada persona no la compone el transcurrir de los acontecimientos, sino el relato que se hace de esos acontecimientos, y en tanto que el propio sujeto es el narrador puede tomar partido en la forma en que se va creando su propio relato.

Este relato, entonces, está dirigido siempre al otro y construido desde los significantes que nos anteceden y no son nuestros (la lengua materna, la cultura...). Por tanto, sin la presencia del otro, no hay historia y no hay proceso de subjetivación. Es así como el otro se hace presente en nuestra experiencia, cuando media en la construcción de un saber encarnado, cuando su presencia genera un vacío, un nuevo espacio por hacer que obliga a pensarse, nombrarse y, en última instancia, recrearse (Piussi y Mañeru, 2006).

Es por esto que Atkinson (2015) dice que, en lugar de interrelaciones, que presupone una independencia previa de las personas, sería más acertado hablar de intrarrelaciones. Docente, estudiante y conocimiento no son entidades cartesianas independientes, sino que se encuentran en continua intrarrelación y en un cambio continuo que es inmanente a la recreación del saber, los significantes y significados.

Entonces, ¿cómo el maestro se hace presente para el estudiante? ¿Cómo el encuentro físico con el adulto se convierte en una relación educativa, con un docente? Estas serán las preguntas sobre las que trataremos de pensar desde el caso concreto y singular de dos alumnas de educación primaria. Y esta mirada a la relación educativa y la presencia la haremos a través de los gestos docentes.

Pensamos el texto en términos de gestos porque estos no se dejan instrumentalizar ni tecnificar, a la vez que aluden y hacen explícita la dimensión corporal en la que se mueve y construye la relación educativa. Y es que, como las relaciones, los gestos son siempre físicos, pero también simbólicos. Cada gesto nace de un sentir, un deseo, una intencionalidad para con el otro. Una caricia, por ejemplo, el contacto de una mano con la del otro tiene un significado radicalmente distinto si lo hace un amante, un padre o un maestro.

La relación educativa nace, entonces, en un encuentro que al ser físico se constituye desde gestos físicos significantes; y es a través de ellos que se va construyendo la relación simbólica de los dos cuerpos en relación. En palabras de Van Manen (1998, p. 189):

Ni siquiera podríamos darnos cuenta cómo los gestos y las palabras se funden en el espacio interpersonal entre el profesor y los alumnos. La palabra es gesto, y el gesto es palabra. Y es mediante este lenguaje como se configura la realidad compartida [...] la calidad gestual funciona dejando a los alumnos entrar con naturalidad en la realidad significativa de la física, la literatura y el arte. A través de los gestos los estudiantes se fijan corporalmente en el terreno de las significaciones a las que los gestos dan vida. 
DIEGO MARTÍN-ALONSO, NIEVES BLANCO Y EDUARDO SIERRA LA PRESENCIA PEDAGÓGICA EN LA CONSTRUCCIÓN DE LA RELACIÓN EDUCATIVA. EL CASO DE UNA MAESTRA DE EDUCACIÓN PRIMARIA

\section{ENFOQUE Y DISEÑO METODOLÓGICO}

Como adelantamos en la introducción, el foco del texto es pensar sobre el lugar simbólico (y también físico) del docente en la relación educativa. En concreto, nos proponemos indagar en las experiencias y procesos a través de los cuales maestras y maestros se hacen presentes para sus estudiantes. Este ejercicio de investigación sobre los significados de la experiencia vivida nos mueve a abordar el estudio desde el marco de la fenomenología hermenéutica, que Van Manen (2003, p. 29) describe como: «el estudio de los significados vividos o existenciales; [la fenomenología hermenéutica] pretende describir e interpretar estos significados hasta un cierto grado de profundidad y riqueza».

La fenomenología hermenéutica nos reclama abordar la investigación desde la preocupación por mantenernos apegados a la experiencia, resistiendo a la tentación por categorizarla o conceptualizarla. Más que un método o teoría de investigación es, en definitiva, una disposición de investigación. Esta actitud fenomenológica termina dando forma y concretándose tanto en los procedimientos de investigación como en el proceso de análisis y escritura del informe final.

En el caso particular de este estudio, los procedimientos de investigación han sido la observación de cerca (Van Manen, 2003) y las conversaciones hermenéuticas (Martín-Alonso, 2019; Sierra y Blanco, 2017). Así, durante dos cursos escolares (2017/18 y 2019/20) hemos acompañado semanalmente a una maestra de un colegio público de la ciudad de Málaga, Clara, y varios de sus estudiantes. En concreto en este texto nos centramos en la experiencia de dos alumnas, Alisha y Hanna, que tenían 10 años al finalizar el trabajo de campo. Durante este tiempo hemos realizado un total de 25 registros de observación con ellas, así como 10 conversaciones hermenéuticas con la maestra y 2 con las alumnas (estas tuvieron lugar en el segundo curso en que acudimos a la escuela).

Por otro lado, para el análisis de textos fenomenológicos, Van Manen (2003) propone inventar una aproximación a partir de los planteamientos de trabajos fenomenológicos previos. En concreto en este texto, dadas las preguntas y foco de la investigación, hemos elaborado nuestra propia aproximación a partir de dos propuestas que hace el autor:

- Análisis exegético. Se trata de poner el propio texto en diálogo con textos de otros autores, buscando una interpretación crítica y completa de estos. Es decir, el enfoque exegético «se organiza en términos de una discusión de dichos textos y de los temas estructurales que sus autores ya han identificado y discutido». (ibíd., p. 187).

- Mediante ejemplos. «Consiste en empezar la descripción haciendo visible la naturaleza esencial del fenómeno y luego completar la descripción inicial mediante la variación sistemática de los ejemplos». (ibíd.). Lo que se persigue con ello es que la variación de ejemplos, y su estudio, nos permita 
DIEGO MARTÍN-ALONSO, NIEVES BLANCO Y EDUARDO SIERRA

LA PRESENCIA PEDAGÓGICA EN LA CONSTRUCCIÓN DE LA RELACIÓN EDUCATIVA.

EL CASO DE UNA MAESTRA DE EDUCACIÓN PRIMARIA

destapar aspectos esenciales de la experiencia que se estudia, en nuestro caso la experiencia de la relación educativa.

Lo que hacemos, por tanto, es pensar en la relación educativa y el lugar que ocupa el docente en ella a partir de la obra de varios autores. Estos textos los ponemos en diálogo con la narración de experiencias concretas que hemos vivido junto a Alisha y Hanna, en diferentes momentos y espacios; de modo que esta "Variación" nos permita vislumbrar algunos aspectos estructurales de la relación educativa capaces de profundizar en la comprensión de un fenómeno tan complejo como necesario.

\section{Hallazgos Y discusión}

\subsection{Invitar}

9 de octubre de $2017^{5}$

Hanna y Alisha tienen, de 8 años, están en $3^{\circ}$ de Primaria y llegaron a España casi al mismo tiempo, en la primavera de 2017. La familia de Hanna viene de Ucrania y la de Alisha de India. Durante el primer mes del curso las ha atendido un maestro de $\mathrm{ATAL}^{6}$ dos veces en semana. Sin embargo, ya que acaban de iniciar el aprendizaje del español, esto no está siendo suficiente para que puedan incorporarse al ritmo de la clase. Por ello, el equipo docente decidió que además del maestro de ATAL, durante los primeros meses debían trabajar varias horas más a la semana en un aula diferenciada con Clara, maestra de refuerzo y apoyo. ${ }^{7}$

Acaba de iniciar la jornada escolar cuando Clara entra al aula del grupo de tercero y, desde la puerta, saluda al tutor, a Hanna y Alisha. Desde allí les explica que van a trabajar juntas para seguir con el estudio del español, y que van a comenzar en ese mismo momento.

Ambas asienten con la cabeza, recogen su material y se vienen con nosotros hasta un aula más pequeña, acondicionada para grupos reducidos. En el centro de esta aula hay una mesa grande. Los cuatro nos sentamos en torno a ella. -¿Qué habéis hecho este fin de semana? — pregunta Clara.

Ambas miran a la maestra, pero ninguna responde. Clara plantea otra pregunta.

5. Todas las escenas son adaptaciones del diario de campo y están escritas en primera persona porque el trabajo de campo lo realizó el autor 1, aunque en su posterior análisis y escritura participaron todos los autores.

6. Maestro de ATAL (Aula Temporal de Adaptación Lingüística), se ocupa de apoyar al tutor e iniciar en el aprendizaje de la lengua española al alumnado inmigrado de países de habla no hispana.

7. A pesar del recelo pedagógico que nos crea esta terminología, lo utilizamos en el artículo por ser la forma tradicional de nombrarlo en el contexto escolar. 
DIEGO MARTÍN-ALONSO, NIEVES BLANCO Y EDUARDO SIERRA LA PRESENCIA PEDAGÓGICA EN LA CONSTRUCCIÓN DE LA RELACIÓN EDUCATIVA. EL CASO DE UNA MAESTRA DE EDUCACIÓN PRIMARIA

-¿A qué habéis jugado?

El objetivo de esas sesiones es acercarse al español a través del lenguaje escrito, pero, sobre todo, de forma oral, de manera que puedan mantener una conversación, seguir las propuestas de clase y hablar con sus compañeros, por lo que entiendo (algo que Clara me corrobora cuando hablo después con ella) que estas preguntas no son meras formalidades para dar la bienvenida a la clase, sino el propósito mismo de la clase: hablar en español. El intento de Clara es que escuchen, comprendan lo que les dicen y formulen una respuesta, iniciando así una conversación.

El silencio de las alumnas se mantiene, y Clara sigue probando a charlar con otros temas y preguntas, hasta que terminan metiéndose, poco a poco, en una conversación.

-¿Hanna has jugado a la Play?

Las dos alumnas mantienen el mutismo y la mirada fija en la maestra.

- ¿Qué has comido el fin de semana Alisha?

-Bocadillo

-¿De qué?

-Buenas noches

Hanna, que permanecía callada, se ríe, parece que está entendiendo lo que dicen y la confusión de su compañera.

- ¿Hanna un bocadillo puede ser de buenas noches? — pregunta Clara instantáneamente.

Hanna niega con la cabeza

-¿De qué puede ser un bocadillo?

Al no responder ninguna alumna, Clara continúa:

-¿Qué has comido hoy?

-Plátano — responde Hanna.

- ¿Plátano has comido? ¿Y qué más?

-Manzana

- ¿Solo has comido manzana y plátano?

Vuelve el silencio acompañado de la mirada fija. Quizá Hanna haya entendido que la pregunta iba sobre comida y ha respondido con algo que le gusta, algo que ha comido o, quizá, la comida que sabía nombrar en ese momento. Parece que Clara ha sentido algo similar y continúa la invitación a la conversación a través de preguntas para dar posibilidad a las alumnas de hablar, aunque sea de manera escueta.

- ¿Tu padre trabaja en una frutería verdad? - la maestra se dirige ahora a Alisha. Silencio.

- ¿Tu padre trabaja en una frutería? —insiste Clara, ahora vocalizando lentamente. - Sí.

-¿Y tú le ayudas?

- Sí. 
DIEGO MARTÍN-ALONSO, NIEVES BLANCO Y EDUARDO SIERRA

LA PRESENCIA PEDAGÓGICA EN LA CONSTRUCCIÓN DE LA RELACIÓN EDUCATIVA.

EL CASO DE UNA MAESTRA DE EDUCACIÓN PRIMARIA

- ¿Y qué vende?

-Tomate y fruta.

- ¿Y cuál es tu favorita?

-Pera

-¡Ah! Qué rica. A mí me gusta mucho la naranja ¿a ti te gusta?

-¡No! -contesta Alisha apretando la nariz y el entrecejo, que parece que hubiese sentido el ácido de la fruta en la boca.

Ante esa desvergonzada expresión, Hanna vuelve a reír. El clima empieza a notarse diferente, las niñas relajan la postura y la mirada.

- ¿A ti te gusta, Hanna? - pregunta Clara

-Sí, y el limón también.

La niña asiente con una sonrisa de entusiasmo. Aunque las respuestas sean sucintas, en parte a causa del conocimiento de las niñas de la lengua en que están hablando, comienza a fluir el diálogo. Tras unos minutos hablando sobre el trabajo de sus familiares, Clara pasa a proponerles una actividad de escritura.

Nuestras clases en la universidad comienzan con un rotundo silencio y miradas, igual que la de Hanna, fijas en nosotros. La expectación inicial es máxima. A medida que avanza la clase y empezamos a entrar en relación, el silencio se va rompiendo: un alumno abre el portátil y comienza a teclear, otra cuchichea con la compañera, otros bajan la cabeza al material que tienen en la mesa, una alumna hace una pregunta... Desde la etapa de educación infantil hasta la universidad, el primer día de clase se escucha el mismo silencio. El estudiante acaba de encontrarse con un adulto que dice ser su maestro, su maestra, y ese encuentro genera expectación, curiosidad por la persona que acaba de llegar: ¿quién eres tú?, ¿realmente puedes enseñarme algo valioso?, ¿qué experiencias me ofreces? Es lo que en el argot escolar, entre docentes, se repite casi como un cliché: "te están tanteando".

En la escena narrada, los silencios son tan fuertes que no nos permiten escuchar lo que Hanna piensa y se pregunta. Podríamos arriesgarnos a imaginar que está tratando de descubrir qué hace allí, qué se espera de ella en ese nuevo espacio y decidiendo cómo estar y actuar ante la nueva maestra. Hasta ese momento había pasado dos meses en el aula, haciendo las mismas actividades que sus compañeros, pero a falta del idioma, sin comprender lo que hacía, se limitaba a imitar lo que estos hacían. Por eso mantiene la mirada fija en la maestra, porque sus dudas, seguimos interpretando, se centran en ella: ¿qué quiere que haga? ¿Por qué me repite las mismas preguntas? ¿Por qué tengo que participar ahora si no lo había hecho antes? ¿Realmente está interesada en saber qué he hecho el fin de semana? ¿En qué riesgo me pone decir algo cuando apenas entiendo la pregunta? En nuestro ejercicio de imaginación interpretativa podemos pensar que los repetidos silencios están ocupados por alguna de estas preguntas (y por supuesto también acompañados de la dificultad de responder en una lengua extranjera a la que comienza a tener acceso). 
DIEGO MARTÍN-ALONSO, NIEVES BLANCO Y EDUARDO SIERRA LA PRESENCIA PEDAGÓGICA EN LA CONSTRUCCIÓN DE LA RELACIÓN EDUCATIVA. EL CASO DE UNA MAESTRA DE EDUCACIÓN PRIMARIA

Este silencio resuena con un soniquete propio del contexto educativo. Y es que cuando estamos en otros espacios no se producen los mismos vacíos: nos presentamos, preguntamos por la otra persona, buscamos intereses comunes y la conversación fluye desde que se inicia el encuentro ¿Por qué se genera ese silencio en las aulas? ¿Qué es tan importante para detenerlo todo y atender, con la mirada fija, al nuevo adulto que acaba de entrar?

Tradicionalmente la enseñanza ha sido pensada y vivida como una anticipación controladora del aprendizaje y, en cierta medida, también del deseo (pensado en los códigos de la motivación, que permiten un aparente control externo y dejan al estudiante como un sujeto pasivo, dependiente de las propuestas que le ofrezca el docente). Sin embargo, justo, al contrario, aprender es construir un sentido vital propio de lo que se va experimentando. El aprendizaje nace y está atravesado por el deseo (Bárcena, 2018; Biesta, 2017; Recalcati, 2014); es el deseo lo que «tensa todo nuestro cuerpo y le incita a desplazarse». (D'Hoest, 2011, p. 79) a ponerse en juego de una manera determinada ante lo que acontece.

Como educadores, entonces, tenemos que asumir que no podemos controlar ni el aprendizaje ni el deseo. Al docente se le escapa la experiencia vivida del otro, los significados y sentidos que construye en lo vivido. Es responsable, por tanto, del proceso de enseñanza, pero no del aprendizaje (Biesta, 2009).

Así, la enseñanza comienza no con una imposición de aprendizaje, sino con un ofrecimiento, con una invitación a la que el estudiante puede, o no, unirse. Tienen sentido en este contexto los silencios de Hanna, que parece preguntarse: ¿quién eres?, ¿qué vamos a vivir en este espacio? ¿qué me traes?, ¿qué deseo hacer con eso?, ¿si digo una palabra equivocada me regañarás o reiremos juntas?, ¿qué puedo aprender contigo? Parece que estuviese tratando de ver cuál es la invitación de la maestra y decidiendo en qué manera la acepta y se pone en juego.

La niña no tardará en empezar a responderse a estas preguntas. La maestra acoge con complicidad la primera risa de Hanna: parece que el espacio, al menos, no es hostil. Las niñas continúan probando, diciendo las primeras palabras, que la maestra acoge no como buenas o malas, sino como su palabra. Y se la devuelve en forma de pregunta: «UUn bocadillo puede ser de buenas noches?». Esa pregunta se convierte en un gesto que, interpretamos, las niñas pueden acoger como una invitación. Una invitación a pensar en su palabra, su manera de nombrar el mundo, este nuevo lugar del mundo que habitan, y hacerlo sin juzgarse ni ser juzgadas.

Al hablar de invitación en esta escena nos referimos al gesto de la maestra que le dice a las niñas que está disponible, que se va a esforzar por conocerlas, permitiéndoles sentirse escuchadas, sin juicios, y poder iniciar así una relación de confianza. No se trata entonces solo de estar disponibles (Martín-Alonso, Blanco y Sierra, 2019; Van Manen, 1998), sino también de que el otro sienta esa disposición, que está ahí para acompañarle.

Para que la relación sea educativa, entonces, debe haber un juego de reconocimientos, debe haber una invitación por parte del adulto y una aceptación por parte 
DIEGO MARTÍN-ALONSO, NIEVES BLANCO Y EDUARDO SIERRA

LA PRESENCIA PEDAGÓGICA EN LA CONSTRUCCIÓN DE LA RELACIÓN EDUCATIVA.

EL CASO DE UNA MAESTRA DE EDUCACIÓN PRIMARIA

del estudiante de entrar en esa relación, reconocerle como maestro y comprometerse con la transformación y trascendencia que la experiencia de relación conllevará. En definitiva, «se trata de una relación elegida (y de filiación) que compone una trama en la que hay lugar tanto para la seducción legítima [...] como para la traición declarada». (Bárcena, 2018, p. 78).

\subsection{Estar ahí}

El acontecimiento irrumpe con tanta fuerza que instala, en un primer momento, un lapso de silencio, de secreto. No es sosiego, sino todo lo contrario; el acontecimiento añade inquietud a la existencia, profunda y auténtica inquietud. Lo que hay que hacer es echar a andar: nuestro movimiento irá colocando las cosas, en el ir y volver, en el andar y desandar. (D’Hoest, 2011, p. 81).

Una vez que se acoge la invitación hay que, en palabras de D'Hoest, «echar a andar». Si se habían atrevido a atravesar la inquietud del nuevo encuentro, si habían roto el silencio con la palabra, la maestra debía estar ahí, en presencia, en esa experiencia imprevisible y de desasosiego, acompañándolas en el camino que iniciaban de aprendizaje de una lengua extranjera.

Las niñas habían comenzado a implicarse en esa misma sesión, y en las que siguieron, en las tareas con la maestra. Pero ¿qué debía hacer ella? ¿Dónde colocarse en el camino que empezaban a transitar? ¿Mejor dejarlas andar solas y que puedan tropezar? ¿O sostenerlas y marcarles el camino?

Para pensar en estas preguntas nos ayuda una de las escenas cotidianas que vivimos con ellas varias semanas después:

\section{9 de noviembre de 2017}

Las dos niñas vienen hoy carialegres. Alisha trae un spinner. Parece nuevo. Me lo enseña ufana y, como ya es costumbre, los cuatro nos sentamos alrededor de la mesa central del aula. Después de una breve bienvenida, Clara inicia el trabajo de la sesión.

-Comenzamos. Tenéis que escucharme, pensar en lo que os digo y lo hacéis ¿Lo habéis entendido? - Las niñas asienten al unísono y Clara continúa, ahora mirando a Hanna -Acaríciame.

La niña se acerca a la maestra y posa la mano sobre su hombro.

-Coge la libreta — dice Clara, esta vez dirigiéndose a Alisha.

La niña coge su cuaderno y lo levanta con las dos manos por encima de su cabeza, como si acabase de ganar un trofeo. Al dejarlo sobre la mesa observo que tiene escritas estas acciones, y otras más, por lo que deduzco que han trabajado el día anterior, que yo no vine, con esta actividad. La maestra continúa dando instrucciones a las niñas: «abrázame», "abre la puerta», "siéntate», "toca a Alisha», etc. 
DIEGO MARTÍN-ALONSO, NIEVES BLANCO Y EDUARDO SIERRA LA PRESENCIA PEDAGÓGICA EN LA CONSTRUCCIÓN DE LA RELACIÓN EDUCATIVA. EL CASO DE UNA MAESTRA DE EDUCACIÓN PRIMARIA

Las dos responden con brío y risueñas a todas las indicaciones. Por la sonrisa de su rostro parece un juego, y por la celeridad con la que actúan parece fácil. Entonces Clara les pide que sean ellas quienes den las instrucciones. Aquí se muestran algo dubitativas y, aunque con algunos fallos en la conjugación y acentuación, son capaces de hacerse entender y dar las instrucciones que desean: -Sientáte en el silla.

-Abre el mochila.

-Coge la goma.

En un primer análisis podemos pensar que el cambio en la actividad de ejecutar las instrucciones a formularlas es un gesto meramente didáctico. Está proponiendo a las niñas la tarea más conveniente para acercarse, desde sus conocimientos, a un vocabulario específico y a la conjugación de verbos en imperativo: primero escuchando y comprendiendo las instrucciones y, a continuación, siendo ellas quienes las producen y crean, una tarea de mayor complejidad. Sin embargo, en un análisis más profundo podemos entrever que este gesto viene movido desde una disposición pedagógica a plantearse qué es lo más adecuado en cada situación y para cada criatura: ¿insistimos en el uso de palabras que parecen saber manejar? ¿Nos adentramos en un vocabulario nuevo? ¿Les ayudo a formular las instrucciones o les dejo perderse y elaborarlas solas? ¿Cómo les ayudo? ¿Qué ayuda necesitan? En definitiva, ¿qué necesitan?

Esta última es una pregunta difícil, si no imposible, de responder pero que debe mantenerse abierta para orientar el trabajo docente (Noddings, 2005). Es la pregunta por lo que necesita el otro y en qué medida me necesita a mí como educador (Gómez, 2018), despertando en la búsqueda de una respuesta el tacto pedagógico (Van Manen, 1998).

Se trata de estar ahí, de situarse a una "distancia respetuosa» (Gusdorf, 2019; Esteve, 2009). Esto significa estar en el lugar adecuado para orientar al otro, acompañarle sin marcarle el camino; es estar lo suficientemente lejos para no imponerse, ni imponer (un conocimiento, la relación...), pero lo suficientemente cerca para que podamos apoyar y orientar en lo que necesite. La manera más gráfica de ejemplificar esto es con el aprendizaje del caminar: cuando el niño se siente cómodo gateando prueba a erguirse. El niño tratará de mantenerse en pie mientras el padre o la madre esté a su lado, le sostenga y compense sus desequilibrios. Cuando estar de pie parezca una aventura asequible probará a dar su primer paso, siempre sujeto y apoyado en el padre, madre. Poco tiempo después podremos ver la peculiar y tradicional escena en que el adulto se pone en cuclillas a dos pasos del niño, con los brazos abiertos e invitándole a llegar caminando hasta él. No tardará mucho en llegar el día en que el adulto repita esto, pero a cinco o seis metros. La madre, el padre, estará valorando dónde debe estar, está midiendo a qué distancia situarse. Ningún sentido tiene ya estar a su vera para que se apoye al levantarse, puede estar de pie sin ayuda y ha aprendido a caer sin dañarse. Tampoco tendría sentido 
situarse en el otro extremo de la habitación, la distancia sería insalvable para la criatura. Ese es el lugar exacto. Debe estar ahí, invitando al niño a dirigirse a él y emprender su camino.

En este sentido, señala Gusdorf (ibíd., p. 118) que «el maestro debe estar atento al acontecimiento; debe hacer preguntas y a veces debe sugerir las respuestas, permaneciendo siempre a una respetuosa distancia.». Esto es precisamente lo que vemos en la última escena narrada. La maestra comienza con una tarea ("tenéis que escucharme, pensar en lo que os digo y lo hacéis») y, cuando esto es asequible, pasa a la siguiente ("ahora os toca a vosotras»). El paso del trabajo de ejecución al de creación de las instrucciones, aunque en apariencia trivial por estar envuelto en lo cotidiano del aula, esconde un profundo sentido pedagógico y una preocupación siempre latente de la educadora por lo que necesitan las niñas a cada momento y cómo situarse ante esas necesidades. Esto significa que la didáctica y la pedagogía no son asuntos independientes, el quehacer en el aula tiene lugar desde una disposición e inquietudes pedagógicas determinadas. Lo didáctico está atravesado por lo pedagógico, de lo contrario se reduce a mera aplicación técnica e instrucción.

Y es que el propósito último de enseñanza no es el propio encuentro con la maestra, sino que este encuentro medie en el acceso a la cultura, a la palabra, así como en el proceso de subjetivación del alumnado (Biesta, 2017). Ese es el cometido del educador, estar ahí, poner su palabra, su cuerpo, su presencia, en relación con el estudiante y mediando en la transmisión del deseo por el saber. La responsabilidad del estudiante será entonces la de crear su propia construcción en la relación con el maestro. Por eso dice Bárcena (2018, p. 101) que «quien recita la lección del maestro, le traiciona.". No se trata de "pensar como el maestro", sino de que la encarnación del conocimiento que es el maestro se convierta en testimonio. La labor del estudiante es, entonces, heredar aquel testimonio del que es testigo. El discípulo es un heredero, en el sentido en que lo plantea Recalcati (2014, p. 40): "Heredar no es solo recibir un sentido del mundo, sino que es también la posibilidad de abrir nuevos sentidos del mundo, nuevos mundos de sentido".

¿Cómo abre la herencia nuevos mundos de sentido? Recalcati (2014, pp. 134-135) sigue su argumentación señalando que se construye desde y en la palabra del otro. Y así, continúa afirmando que

No somos más que el conjunto estratificado de todas las huellas, las impresiones, las palabras, los significantes que, procedentes del Otro, nos han formado. No podemos hablar de nosotros mismos sin hablar de los Otros, de todos los Otros que han determinado, fabricado, producido, marcado, plasmado nuestra vida.

Es por ello por lo que no resulta baladí la tarea que narramos (y que es representación de muchas otras que se repitieron a lo largo del curso). La maestra les está dando, literal y simbólicamente, la palabra. Les da la posibilidad de nombrar, nombrarse y estar en relación con sus compañeros a través de una palabra - y una cultura- que puede ser compartida. 
DIEGO MARTÍN-ALONSO, NIEVES BLANCO Y EDUARDO SIERRA LA PRESENCIA PEDAGÓGICA EN LA CONSTRUCCIÓN DE LA RELACIÓN EDUCATIVA. EL CASO DE UNA MAESTRA DE EDUCACIÓN PRIMARIA

\subsection{Retirarse}

\section{9 de noviembre de 2019}

Hanna está todo el horario lectivo con su grupo, ya no son necesarios esos espacios específicos con el maestro de ATAL ni con Clara.

Es hora de matemáticas. La tutora del grupo, María, saca del armario una caja con regletas de Cuisenaire. Mientras reparte el material, María va introduciendo a los niños en la tarea y recordando lo que hicieron los días anteriores.

-¿Quién me dice un múltiplo de tres?

-Seis — responde Mónica con celeridad, sin dejar tiempo a sus compañeros a responder.

$-¿$ Y un múltiplo de siete?

-Catorce — quien se adelanta ahora es Carlos.

-¿Por qué es un múltiplo de siete?

-Porque si multiplicas siete por dos da catorce — responde el niño con ímpetu. -A ver si sabéis representar eso con las regletas. Pensad en un número y haced luego múltiplos de ese número.

La tarea se antoja sencilla para algunos estudiantes, que se sumergen con decisión en ella. Pero no todos parecen saber cómo proceder. Entre ellos está Hanna, que mira expectante a sus compañeros, parece que tratando de averiguar qué hacer. Entonces se levanta y se dirige a la maestra.

-¿Qué significa representar con las regletas? — pregunta la niña.

La maestra le pone la mano en el hombro y juntas se dirigen a su mesa. Allí le vuelve a explicar la tarea. Cuando María se aleja, Hanna devuelve la mirada a sus regletas y no la despega hasta que termina el ejercicio, unos minutos después. -El último paso es ponerlo en el cuaderno -interviene María entre el bullicio de la clase. Esto significa que deben representar de forma gráfica y numérica las composiciones que han hecho con las regletas.

Cada quien va a su ritmo. Hanna, que ya ha terminado todo, va a la mesa de Mohamed, que parece algo perdido, pero termina entendiendo la tarea con las explicaciones de su compañera, por lo que la niña cambia de lugar. Ahora se mueve hasta la mesa de Alejandro. Se agacha para ponerse a su altura, pues él está sentado. Sonríen. No sé de qué hablan. Me acerco para poder escucharles. Hanna está explicándole cómo se hacen los múltiplos con las regletas.

-¡No tienes que copiarlo! ¡Tienes que entenderlo! —exclama risueña Hanna cuando se percata de que su compañero estaba imitando arbitrariamente lo que ella hacía. Sigue ayudándole, entre risas, a terminar la tarea.

Dos años después, Hanna se mueve por el aula con una cierta independencia, comprende lo que le dicen y, aunque todavía con equívocos, puede conversar de forma fluida. Sus maestras han dado «un paso atrás»: Clara ya no aparece en el día a día de la alumna y su nueva tutora le da un cierto espacio, acercándose solo 
cuando le reclama. Aunque la protagonista de la última escena es Hanna, su tutora tiene una presencia estructural en la "trama». Es quien crea el contexto del aula y acompaña a la niña en la medida en que lo necesita. Así, por ejemplo, cuando Hanna y Alejandro ríen a propósito de la actividad, María no aparece, pero está. Se encuentra a cierta distancia, se ha retirado hasta un lugar adecuado que permitiese a la niña comprender la tarea y ayudar a su compañero. De suceder lo contrario, si hubiese estado pegada a Hanna, por ejemplo, para explicarle el significado de cada palabra y cómo se conjugan los verbos que esté utilizando, el final de la escena no habría sido de la misma manera y los chicos no habrían podido ayudarse y construir la solución por sí mismos.

Y es que, si la relación es educativa, el alumnado irá creciendo y esa distancia respetuosa de la que hablamos tenderá a dilatarse. Si queremos enseñar al niño a andar, cada vez necesitará menos nuestra ayuda, nuestra presencia. El proceso de emancipación ${ }^{8}$ (Biesta, 2010, 2017) pasa a una nueva etapa y el educador tiene que retirarse. La relación, si es educativa, está abocada a la separación. De hecho, debe anhelar el momento en que esta llegue, pues su propósito último es que el niño, niña, camine por sí mismo y no nos necesite. Este fin último de la relación educativa lo sintetiza Van Manen (1998, p. 72) en una suerte de paradoja: "Los niños necesitan apoyo para llegar a ser independientes».

Aquí retirarse no significa que la relación desaparezca. La ausencia de presencia física no significa tampoco que la relación ya no exista. La relación perdura, pero cambia la naturaleza de la presencia. Con el devenir de la relación y el crecimiento del alumnado son necesarios e ineludibles cambios físicos (puede pasar, como le sucede a Hanna, de sentarse a su vera en un aula solo para ella a deambular en un aula llena de estudiantes), temporales (las visitas de Clara pasaron de ser diarias a esporádicas y, con el paso del curso, a desaparecer) y simbólicos (cómo le acompaña, cómo cuestiona lo que hace, etc.).

Esta separación no significa romper el vínculo, justo lo contrario, puede que la separación sea una necesidad y exigencia del niño o niña, de mantenerse en relación, pero a una cierta distancia. Esto es, precisamente, lo que le sucedió a Hanna. En una conversación que mantuvimos con ella, nos comentaba su deseo de hacer sola la tarea:

AUTOR 1: Si viene hoy un profe nuevo a clase ¿qué tendría que contarle de ti? HANNA: A mí cuando viene un profe nuevo, dile que yo trabaja bien pero un profe me tiene que explicarme a mí para que entienda qué tengo que hacer y después ya lo hago sin problemas.

8. Por emancipación entendemos en este texto la construcción que hace Gert Biesta. A diferencia de la socialización, no se trata de acoger el orden existente, sino de ser un sujeto «independiente de acción» en relación con los demás, en sociedad (Biesta, 2010). La emancipación es «una ruptura que es la aparición de la subjetividad. De esta manera, la emancipación puede interpretarse como un proceso de subjetivación” (Biesta, 2017, p. 104). 
DIEGO MARTÍN-ALONSO, NIEVES BLANCO Y EDUARDO SIERRA LA PRESENCIA PEDAGÓGICA EN LA CONSTRUCCIÓN DE LA RELACIÓN EDUCATIVA. EL CASO DE UNA MAESTRA DE EDUCACIÓN PRIMARIA

$[\ldots]$

AUTOR 1: Yo aquí en clase te veo que trabajas mucho.

HANNA: Estoy aquí dos años, ya lo he aprendido a hacer sola en clase.

AUTOR 1: Pero a veces parece que te cuesta más...

HANNA: El ejercicio puede ser fácil, pero si no entiendo la pregunta no puede hacerlo.

AUTOR 1: ¿Y cuándo no lo entiendes qué haces?

HANNA: Pues si no lo entiendo, yo le digo «seño María no lo entiendo» y ella me lo explica, o le dice a [mi compañero] Carlos, que lo entiende, que me lo explique.

AUTOR 1: ¿Y no prefieres que esté la seño Clara como antes aquí ayudándote? HANNA: Y si el año que viene no está la seño Clara, ¿qué voy a hacer? ¿Me voy a dejar el sexto curso?

Lo que nos dice Hanna, interpretamos, es que una presencia desmesurada le estorba, no le deja crecer, algo que ella vincula en la conversación con la preparación para el próximo curso. Y es que cuando el alumnado acepta la invitación inicial del maestro está comprometiéndose con su propio proceso, movido este por un deseo de crecimiento, de emancipación. Por lo que, paradójicamente, la relación junto al otro se construye desde el deseo de crear un espacio propio, de un ir haciendo por sí mismo (que no a sí mismo). Lo contrario, el anhelo de permanecer siempre discípulo, señala a una falta de deseo de emancipación, en tanto que se permanece siempre dependiente de la presencia del educador. Esto es algo que expone Bárcena (2018, p. 93) con precisión:

Cyrus le dice a su hijo: 'Pronto te irás, ya tienes edad', y esta afirmación entraña una profunda verdad, si es cierto que el acto educativo acarrea consigo el destino de la separación que acompaña la relación educativa (la de los padres con sus hijos, la de los maestros con sus discípulos). Porque no hay educación que no implique una salida al exterior, una separación, cierta clase de ruptura.

Esa «Salida al exterior» nos resuena a la idea de emancipación de la que nos habla Biesta, de manera que el maestro, ante la fragilidad del niño, asume una responsabilidad hacía él (Gómez, 2018). Se trata de la responsabilidad de acompañar en el proceso de emancipación, es decir, «el profesor no es quien empodera a quien no tiene poder, sino solo quien abre el camino para que pueda ejercer un poder que ya tiene consigo". (Biesta, 2017, p. 14). Se trata entonces de cómo introducir a los niños en el mundo, no para repetir lo que ya existe, sino para ayudarles a venir al mundo, que lo hagan suyo y relacionarse con él desde su singularidad (Bárcena y Mèlich, 2000; Biesta, 2016).

Y dado que la emancipación acontece en procesos de subjetivación y que esta solo tiene lugar en relación, la emancipación solo es posible en relación, en un proceso «dialógico» en que educador y estudiante son "Co-sujetos» (Biesta, 2017). 
Por esto decía Atkinson (2015) que existe una suerte de intrarrelación. Pensándolo con las escenas: Hanna no podría moverse con esa libertad por el aula si en su historia no estuviese, entre otras, la relación con Clara; tampoco podría conocer de forma independiente el significado y sentido de las regletas. Solo ha sido a través de la relación que ha accedido al lenguaje, a la palabra, y con ella ha podido salir, literal y simbólicamente, de su espacio y dirigirse al encuentro con sus compañeros. Como concluye Recalcati (2014, p. 135): «Somos nuestra palabra, pero nuestra palabra no existiría si no se hubiera formado a través de la palabra de otros que nos han hablado".

\section{ConCLUSIÓN}

En nuestro análisis hemos presentado tres gestos que aparentan ser secuenciales en el desarrollo de la relación educativa. Sin embargo, en la vivencia de las niñas no podemos atrevernos a pensar que haya sucedido así; somos nosotros, como investigadores, que en un ejercicio de comprensión de estas experiencias concretas necesitamos nombrarlas, encerrarlas en el marco de la palabra para que se nos haga, de alguna manera, tangible el proceso de construcción de la relación educativa. No pretendemos, por tanto, secuencializar o tecnificar la relación educativa, sino pensar sobre ella en el contexto de experiencias singulares.

Hemos observado cómo la maestra trata de "estar ahí» (Praetorius, 2002), actuando desde una deliberación continua que gira en torno a una pregunta: "qqué necesitas?». Y es que, como en el aprendizaje del caminar, cada criatura nos necesita en un lugar diferente. Así, en la relación educativa el docente se responsabiliza por el cuidado del otro, con la esperanza (Van Manen, 1998) de que esa experiencia le ayudará a crecer. Es decir, el propósito del docente es que su experiencia de relación con la niña, con el niño, medie en el acceso y apropiación de la cultura y los conocimientos que se le ofrecen en la escuela, con el fin de que esto le permita construir su propia historia a través de la que vivir en el mundo.

Es en este sentido que la investigación fenomenológico-hermenéutica es un acto político, en tanto que cuestiona el sentido de los programas curriculares y la escuela misma, al ponerla en relación con la experiencia vivida por el alumnado y cómo esta le mueve a construir un lugar propio en la comunidad. Las preguntas que nos hacíamos por la naturaleza de la relación educativa y, en particular, por la experiencia de Hanna y Alisha, nos han permitido entrever cómo es en la mediación del educador (y no en el diseño y aplicación técnica de programas homogéneos) donde se accede a la palabra, a la cultura, al mundo.

\section{REFERENCIAS BIBLIOGRÁFICAS}

Atkinson, D. (2015). The adventure of pedagogy, learning and the not-known. Subjectivity, 8(1), 43-56. https://doi.org/10.1057/sub.2014.22 
DIEGO MARTÍN-ALONSO, NIEVES BLANCO Y EDUARDO SIERRA LA PRESENCIA PEDAGÓGICA EN LA CONSTRUCCIÓN DE LA RELACIÓN EDUCATIVA. EL CASO DE UNA MAESTRA DE EDUCACIÓN PRIMARIA

Arbiol, C. (2018). Elementos para una pedagogía de la alteridad en la práctica de educadoras sociales. Un estudio narrativo. Teoría de la Educación. Revista Interuniversitaria, 30(2), 109-129. https://doi.org/10.14201/teoredu3021109129

Arnaus, R. (2013). La relación como práctica política en la formación inicial de educadoras y educadores sociales. Revista Interuniversitaria de Formación del Profesorado, 78(23), 71-88.

Ayala, R. (2018). La relación pedagógica: En las fuentes de la experiencia educativa con van Manen. Revista Complutense de Educación, 29(1), 27-41. https://doi.org/10.5209/ RCED. 51925

Bárcena, F. (2012). Una pedagogía de la presencia. Crítica filosófica a la impostura pedagógica. Teoría de la Educación. Revista Interuniversitaria, 24(2), 25-57. https://revistas.usal.es/ index.php/1130-3743/article/viewFile/10354/10793

Bárcena, F. (2018). Maestros y discípulos. Anatomía de una relación. Teoría de la Educación. Revista Interuniversitaria, 30(2), 73-108. https://doi.org/10.14201/teoredu30273108

Bárcena, F., y Mèlich, J. C. (2000). La educación como acontecimiento ético: Natalidad, narración y hospitalidad. Paidós Ibérica.

Biesta, G. (2009). Good education in an age of measurement: On the need to reconnect with the question of purpose in education. Educational Assessment, Evaluation and Accountability, 21(1), 33-46. https://doi.org/10.1007/s11092-008-9064-9

Biesta, G. (2010). A new logic of emancipation: The methodology of Jacques Rancière. Educational theory, 60(1), 39-59. https://doi.org/10.1111/j.1741-5446.2009.00345.x

Biesta, G. (2011). Aprendiz, estudiante, hablante. ¿Por qué importa cómo llamamos a quienes enseñamos? En J. Masschelein \& J. Larrosa (Eds.), Jacques Rancière. La educación pública y la domesticación de la democracia (pp. 150-174). Miño y Dávila.

Biesta, G. (2016). Reconciling ourselves to reality: Arendt, education and the challenge of being at home in the world. Journal of Educational Administration and History, 48(2), 183-192. https://doi.org/10.1080/00220620.2016.1144580

Biesta, G. (2017). El bello riesgo de educar. SM.

Cima, R. (2012). Pedagogía cultural para una educación hacia el encuentro. RUEDES, 3, 1-17. https://core.ac.uk/reader/61886310

Clandinin, D. J. (2013). Engaging in narrative inquiry. Left Coast Press.

Clandinin, D. J., \& Connelly, F. M. (1995). Teachers' professional knowledge landscapes. Teachers College Press.

Contreras, J. (2016). Tener historias que contar: Profundizar narrativamente la educación. Roteiro, 41(1), 15-40. https://doi.org/10.18593/r.v41i1.9259

D'Hoest, F. (2011). Cierta experiencia de la distancia en educación: Sobre «El hijo» de los Dardenne. Bajo Palabra, 6, 73-82. https://revistas.uam.es/bajopalabra/article/view/3349

Esteve, J. M. (2009). La urdimbre de la relación educativa. Presentado en XXVIII Seminario Interuniversitario de Teoría de la Educación, Oviedo. http://redsite.es/docu/28site/ Jose\%20Manuel\%20Esteve\%20Zarazaga.pdf

Gómez, D. (2018). Fragilidad y hostilidad. Los gestos pedagógicos del encuentro con el otro. Universitat de Barcelona.

Gusdorf, G. (2019). ¿Para qué profesores? Por una Pedagogía de la Pedagogía. Miño y Dávila. Presentación: Fernando Bárcena. Traducción y notas: Fernando Fuentes Megías. 
DIEGO MARTÍN-ALONSO, NIEVES BLANCO Y EDUARDO SIERRA

LA PRESENCIA PEDAGÓGICA EN LA CONSTRUCCIÓN DE LA RELACIÓN EDUCATIVA.

EL CASO DE UNA MAESTRA DE EDUCACIÓN PRIMARIA

Jordán, J. A. (2011). Disposiciones esenciales de los profesores en las relaciones con sus alumnos desde una perspectiva ética-pedagógica. Educación XX1, 14(1), 59-87. https:// doi.org/10.5944/educxx1.14.1.263

Martín-Alonso, D. (2019). El tejido curricular. Indagación narrativa sobre la relación educativa y el proceso de creación curricular. Universidad de Málaga. https://hdl.handle. net/10630/19311

Martín-Alonso, D., Blanco, N., y Sierra, J. E. (2018). Indagación narrativa sobre las tensiones vividas por un profesor de educación secundaria en el proceso de creación curricular. Archivos Analíticos de Políticas Educativas, 26. https://doi.org/10.14507/epaa.26.3571

Martín-Alonso, D., Blanco, N., y Sierra, J. E. (2019). Comprensión pedagógica y construcción de la relación educativa. Una indagación narrativa. Teoría de la Educación. Revista Interuniversitaria, 31(1), 103-122. https://doi.org/10.14201/teri.19442

Molina, M. D., Blanco, N., y García, S. (2013). El profesorado y «su lugar» para estudiantes de Bachillerato. Hallazgos de una investigación sobre trayectorias de éxito escolar. Educaçao, 38(2), 265-276. https://doi.org/10.5902/198464447726

Noddings, N. (2005). Identifying and responding to needs in education. Cambridge Journal of Education, 35(2), 147-159. https://doi.org/10.1080/03057640500146757

Pinar, W. F., y Irwin, R. L. (2004). Curriculum in a new key: The collected works of Ted T. Aoki. Routledge.

Piussi, A. M., y Mañeru, A. (Eds.). (2006). Educación, nombre común femenino. Octaedro.

Praetorius, I. (2002). La filosofía del saber estar ahí. Para una política de lo simbólico. Duoda, Revista de Estudios Feministas, 23, 99-110. http://revistasacademicas.ucol.mx/index.php/ generos/article/view/1084/pdf

Recalcati, M. (2014). El complejo de Telémaco. Padres e hijos tras el ocaso del progenitor. Anagrama.

Recalcati, M. (2016). La hora de clase: Por una erótica de la enseñanza. Anagrama.

Rodgers, C., \& Raider-Roth, M. B. (2006). Presence in teaching. Teachers and Teaching, 12(3), 265-287. https://doi.org/10.1080/13450600500467548

Sierra, J. E., y Blanco, N. (2017). El aprendizaje de la escucha en la investigación educativa. Qualitative Research in Education, 6(3), 303-326.

Van Manen, M. (1998). El tacto en la enseñanza: El significado de la sensibilidad pedagógica. Paidós.

Van Manen, M. (2003). Investigación educativa y experiencia vivida: Ciencia bumana para una pedagogía de la acción y la sensibilidad. Idea Books.

Vila Merino, E. (2019). Repensar la relación educativa desde la pedagogía de la alteridad. Teoría de la Educación. Revista Interuniversitaria, 31(2), 177-196. https://doi.org/10.14201/ teri. 20271 